\title{
Interactions Between Caveolin-1 (rs3807992) Polymorphism and Major dietary patterns on Cardio-metabolic Risk Factors Among Obese and Overweight Women
}

\author{
Faezeh Abaj ${ }^{1}$, Fariba Koohdani ${ }^{1}$, Masoumeh Rafiee ${ }^{1}$, Mir saeed Yekaninejad ${ }^{1}$, and \\ Khadijeh Mirzaei ${ }^{1}$ \\ ${ }^{1}$ Affiliation not available
}

January 4, 2021

\begin{abstract}
Background: Caveolin is a cholesterol-dependent essential component located in caveolae.

Several studies have been shown CAV-1 SNP related to cardio-metabolic parameters in animal

models, however there is few studies in humans. Importantly, there is no study has investigated

the interaction between CAV-1 rs3807992 gene and dietary pattern on CVDs risk factors in Iranian

population.

Methods: The current cross-sectional study was conducted on 404 overweight and obese females with mean age of 36 years. Dietary intake obtained from FFQ with 147 items. The CAV-1

genotype was measured by the PCR-RFLP method. The anthropometric measurements, serum lipid profile and inflammatory markers were measured.

Results: There was a significant interaction between CAV-1 rs3807992 and healthy dietary pattern on HDL ( $\mathrm{P}$ interaction=0.03), TC/HDL $(\mathrm{P}$ interaction=0.03) and hs-CRP ( $\mathrm{P}$ interaction=0.04); in A-allele carriers, higher adherence to the healthy dietary pattern was related to higher level of HDL and lower TC/HDL and hs-CRP. As well as, the significant interactions were observed between CAV-1 rs3807992 and unhealthy dietary pattern in relation to TG (P interaction $=0.001)$, AST $(\mathrm{P}$ interaction $=0.01)$ and $\mathrm{MCP}-1(\mathrm{P}$ interaction $=0.01) ; \mathrm{A}$-allele carriers were more adherence to the unhealthy dietary pattern to lower levels of TG, AST and MCP-1.

Conclusions: Our study showed that CAV-1 rs3807992 SNP interacts with adherence to unhealthy or healthy dietary patterns to influence several cardio-metabolic risk factors in obese and overweight females. Further large prospective studies are warranted to confirm our findings
\end{abstract}

